\title{
Intravenous versus Oral Acetaminophen for Pain: Systematic Review of Current Evidence to Support Clinical Decision-Making
}

\author{
Farah Jibril, Sherif Sharaby, Ahmed Mohamed, and Kyle J Wilby
}

\begin{abstract}
Background: Intravenous (IV) acetaminophen is increasingly used around the world for pain control for a variety of indications. However, it is unclear whether IV administration offers advantages over oral administration.

Objective: To identify, summarize, and critically evaluate the literature comparing analgesic efficacy, safety, and pharmacokinetics for IV and oral dosage forms of acetaminophen.

Data Sources: A literature search of the PubMed, Embase, and International Pharmaceutical Abstracts databases was supplemented with keyword searches of Science Direct, Wiley Library Online, and Springer Link databases for the period 1948 to November 2014. The reference lists of identified studies were searched manually.

Study Selection and Data Extraction: Randomized controlled trials comparing IV and oral dosage forms of acetaminophen were included if they assessed an efficacy, safety, or pharmacokinetic outcome. For each study, 2 investigators independently extracted data (study design, population, interventions, follow-up, efficacy outcomes, safety outcomes, pharmacokinetic outcomes, and any other pertinent information) and completed risk-of-bias assessments.

Data Synthesis: Six randomized clinical trials were included. Three of the studies reported outcomes pertaining to efficacy, 4 to safety, and 4 to pharmacokinetics. No clinically significant differences in efficacy were found between the 2 dosage forms. Safety outcomes were not reported consistently enough to allow adequate assessment. No evidence was found to suggest that increased bioavailability of the IV formulation enhances efficacy outcomes. For studies reporting clinical outcomes, the results of risk-of-bias assessments were largely unclear.

Conclusions: For patients who can take an oral dosage form, no clear indication exists for preferential prescribing of IV acetaminophen. Decision-making must take into account the known adverse effects of each dosage form and other considerations such as convenience and cost. Future studies should assess multiple-dose regimens over longer periods for patients with common pain indications such as cancer, trauma, and surgery.
\end{abstract}

Keywords: acetaminophen, paracetamol, intravenous, analgesia, pain

Can J Hosp Pharm. 2015;68(3):238-47

\section{RÉSUMÉ}

Contexte : L'administration intraveineuse d'acétaminophène est de plus en plus employée partout dans le monde pour combattre la douleur due à toute une gamme de causes. Cependant, on ignore si elle offre des avantages comparativement à l'administration d'acétaminophène par voie orale.

Objectif: Relever, résumer et faire une évaluation critique de la littérature qui compare l'efficacité analgésique, la sécurité et le comportement pharmacocinétique des formes pharmaceutiques intraveineuse et orale d'acétaminophène.

Sources des données : Une recherche documentaire dans les bases de données de PubMed, Embase et International Pharmaceutical Abstracts a été complétée à l'aide de recherches de mots clés dans les bases de données de Science Direct, Wiley Library Online et Springer Link entre 1948 et novembre 2014. Un examen des bibliographies des études retenues a été réalisé manuellement.

Sélection des études et extraction des données : Les essais cliniques à répartition aléatoire comparant les formes pharmaceutiques intraveineuse et orale d'acétaminophène ont été inclus lorsqu'ils évaluaient des résultats sur l'efficacité, la sécurité ou la pharmacocinétique. Pour chaque étude, deux chercheurs travaillant de façon indépendante ont extrait des données (sur le plan de l'étude, la population, les interventions, le suivi, l'efficacité, la sécurité, la pharmacocinétique et sur toute autre information pertinente) et ils ont rempli des évaluations du risque de biais.

Synthèse des données : Au total, six essais cliniques à répartition aléatoire ont été retenus aux fins de l'analyse. Parmi ceux-ci, trois présentaient des résultats sur l'efficacité, quatre sur la sécurité et quatre sur la pharmacocinétique. Aucune différence cliniquement significative quant à l'efficacité n'a été relevée entre les deux formes pharmaceutiques. Les résultats sur la sécurité n'étaient pas présentés assez systématiquement pour permettre une évaluation pertinente. Aucune donnée n'a été trouvée permettant de croire que la biodisponibilité accrue de la préparation intraveineuse augmente l'efficacité. Pour les études présentant des résultats cliniques, le bilan des évaluations du risque de biais était en grande partie équivoque.

Conclusions : Il n'y a pas d'indication claire favorisant la prescription d'acétaminophène par voie intraveineuse chez les patients en mesure de prendre la forme pharmaceutique orale. Tout choix doit tenir compte des effets indésirables propres à chaque forme pharmaceutique ainsi que d'autres facteurs, notamment la commodité et le coût. Des études ultérieures devraient évaluer les schémas à dose multiples sur une plus longue période chez les patients souffrant de douleurs courantes telles que celles causées par un cancer, un trauma ou une chirurgie.

Mots clés : acétaminophène, paracétamol, administration intraveineuse, analgésie, douleur 


\section{INTRODUCTION}

A cetaminophen (paracetamol) is recognized as one of the most commonly used synthetic, nonopioid, centrally acting analgesic agents. It represents a key part of pain management in patients with cancer, and is used preoperatively, intraoperatively, and postoperatively in a wide range of surgical settings, offering effective and fast pain relief. ${ }^{1-4}$ Acetaminophen has a well-established efficacy profile, favourable adverse drug reaction profile, and very low potential for harmful drug-drug interactions. ${ }^{5}$

Acetaminophen has been available in oral and rectal formulations for decades. However, controversy exists regarding the suitability of these formulations for use in some settings, such as postoperative or acute care. ${ }^{6,7}$ Intravenous (IV) acetaminophen was first commercialized in Europe, in 2002. Later, in 2010, the US Food and Drug Administration approved an IV formulation of acetaminophen for management of mild to moderate pain, management of moderate to severe pain with adjunctive opioid analgesics, and reduction of fever in adults and children 2 years and older. ${ }^{8}$ Since then, acetaminophen has become one of very few nonopioid analgesics available in oral, rectal, and IV formulations. ${ }^{9}$

The efficacy of IV administration of acetaminophen relative to placebo was recently established in a pooled analysis of randomized controlled trials assessing patient satisfaction with acute postoperative pain control. ${ }^{10}$ The 5 trials that were included rated patient satisfaction on a 4-point scale $24 \mathrm{~h}$ after dosing. The analysis showed that patients who received IV acetaminophen reported excellent satisfaction with pain control more often than those who received placebo (32.3\% versus $15.9 \%)$. IV acetaminophen was the strongest predictor of excellent patient satisfaction in a multivariate analysis (odds ratio [OR] 2.76, 95\% confidence interval [CI] 1.81 to 4.23). Therefore, compared with placebo, IV acetaminophen was deemed efficacious for patients presenting with acute pain. ${ }^{10}$

Other considerations have deemed the IV route for administration of acetaminophen advantageous in certain situations, such as when the oral or rectal route is unsuitable or ineffective (e.g., because of emesis) or when the high variability in bioavailability with rectal administration is unacceptable for a particular patient. ${ }^{8,9,11}$ IV administration has become the route of choice for rapid analgesia in inpatient and postoperative settings, largely because of evidence suggesting it may reduce the need for other analgesics such as opioids..$^{2,10,12,13}$ One of the main clinical and practical advantages associated with IV administration is the faster onset of analgesia relative to an equivalent oral dose. In addition, IV administration is associated with more predictable pharmacokinetic and pharmacodynamic behaviour. ${ }^{4,7,12,14}$ Another potential advantage of IV administration of acetaminophen is avoidance of first-pass hepatic exposure through the portal circulation, which may reduce the potential for hepatic injury. ${ }^{15-18}$

Despite the advantages offered by IV administration of acetaminophen, the choice of this route over oral administration should also take into account associated risks and inconveniences. Indeed, the risks associated with IV administration of most drugs include infection, phlebitis, and local irritation. Furthermore, the time needed for IV drug administration, the inconvenience to patients, and the increased direct and indirect costs suggest that the most suitable route of administration should be carefully selected for each patient. This is especially true in situations where the clinician may believe that IV therapy is better or faster-acting than oral formulations. In particular, the IV formulation of acetaminophen has a higher cost and longer administration time (15 min) than oral forms, ${ }^{19}$ factors that must be considered during therapeutic decision-making.

It is currently unclear whether IV administration of acetaminophen is more effective and equally safe compared to oral administration for patients with pain. The objective of this systematic review was to identify, summarize, and critically evaluate the literature comparing analgesic efficacy, safety, and pharmacokinetics for IV and oral dosage forms of acetaminophen.

\section{METHODS}

A literature search was completed using the databases PubMed (1948 to January 2015), EMBASE (1980 to January 2015), and International Pharmaceutical Abstracts (1970 to January 2015), supplemented with keyword searches of Science Direct, Wiley Library Online, and Springer Link databases. Databases were searched using the keywords "acetaminophen" OR "paracetamol" combined with "intravenous" OR "infusion" OR “injection”, and "pain” OR "analgesia” OR "analgesic" using AND to combine search term categories. Keywords were searched as free text in International Pharmaceutical Abstracts but were linked to MeSH terms (Medical Subject Headings) in PubMed and EMBASE, where applicable. The website clinical trials.gov was searched for ongoing or potentially unpublished studies. Included studies were limited to those conducted in humans and published in English. The reference lists of identified studies were manually reviewed to supplement the electronic search methods.

Studies were selected on the basis of the following predefined inclusion criteria: randomized trials in adult humans that reported at least one clinical or pharmacokinetic outcome. Studies were included if they reported outcomes for an IV formulation of acetaminophen and a comparator group that received oral acetaminophen. Studies assessing the prodrug, propacetamol, were excluded because of its non-availability and association with adverse effects that might have biased safety outcomes. Nonrandomized studies and studies that did not include both IV and oral routes were excluded. Outcomes of interest were efficacy outcomes (e.g., pain scores, opioid-sparing, use of as-needed pain medications, length of stay), safety outcomes (e.g., incidence of adverse events or serious adverse events), and pharmacokinetic outcomes (e.g., maximum plasma concen- 
tration [ $C_{\max }$ ], time to maximum plasma concentration [ $T_{\max }$, area under the curve [AUC], and bioavailability). No exclusion criteria were set based on study quality markers.

Data for the main analysis (study design, population, interventions, follow-up, efficacy outcomes, safety outcomes, pharmacokinetic outcomes, and any other pertinent information) were extracted from each study by 2 of the investigators using a standardized extraction tool. Extraction results were cross-examined and assessed for discrepancies, which were resolved by discussion if needed. A third investigator was consulted for discrepancies that could not be resolved by discussion. Data pertaining to risk of bias were extracted according to the Cochrane Collaboration's risk-of-bias assessment tool. ${ }^{20}$ Data extracted for assessment of risk of bias included information about random sequence generation, allocation concealment, blinding, incomplete outcome data, selective outcome reporting, and other biases based on study design or identified confounders. Each component was ranked on a categorical scale for each domain (high risk of bias, low risk of bias, or unclear risk of bias). Two investigators extracted these data from each study and ranked each component. The results were cross-examined and discrepancies resolved according to the methods described above.

\section{RESULTS}

A total of 6 studies met the inclusion criteria and were included in the review. ${ }^{21-26}$ The results of the search strategy, with reasons for exclusions, are given in Figure 1. The characteristics of included studies are summarized in Table 1, with outcome results for each study in Table 2 and risk-of-bias assessments in Table 3. All of the included studies were prospective randomized clinical trials, of which 4 were designed to use parallel groups $^{22-24,26}$ and 2 were designed to use a 3-way randomized crossover method ${ }^{21,25}$ (Table 1).

\section{Studies Reporting Efficacy Outcomes}

Three of the studies identified reported efficacy outcomes. ${ }^{22,24,26}$ Pettersson and others ${ }^{22}$ compared the opioid-sparing effects of oral and IV acetaminophen in the postoperative setting, for patients who had undergone coronary artery bypass grafting. The study drug (acetaminophen, either oral or IV) was given when patients first awakened after surgery, and additional doses were given every $6 \mathrm{~h}$ until 0900 the next morning. The opioid used for rescue analgesia was an IV infusion of ketobemidone, with $8 \mathrm{mg}$ of the IV formulation equalling about $10 \mathrm{mg}$ of IV morphine. The use of opioids was significantly lower in the group

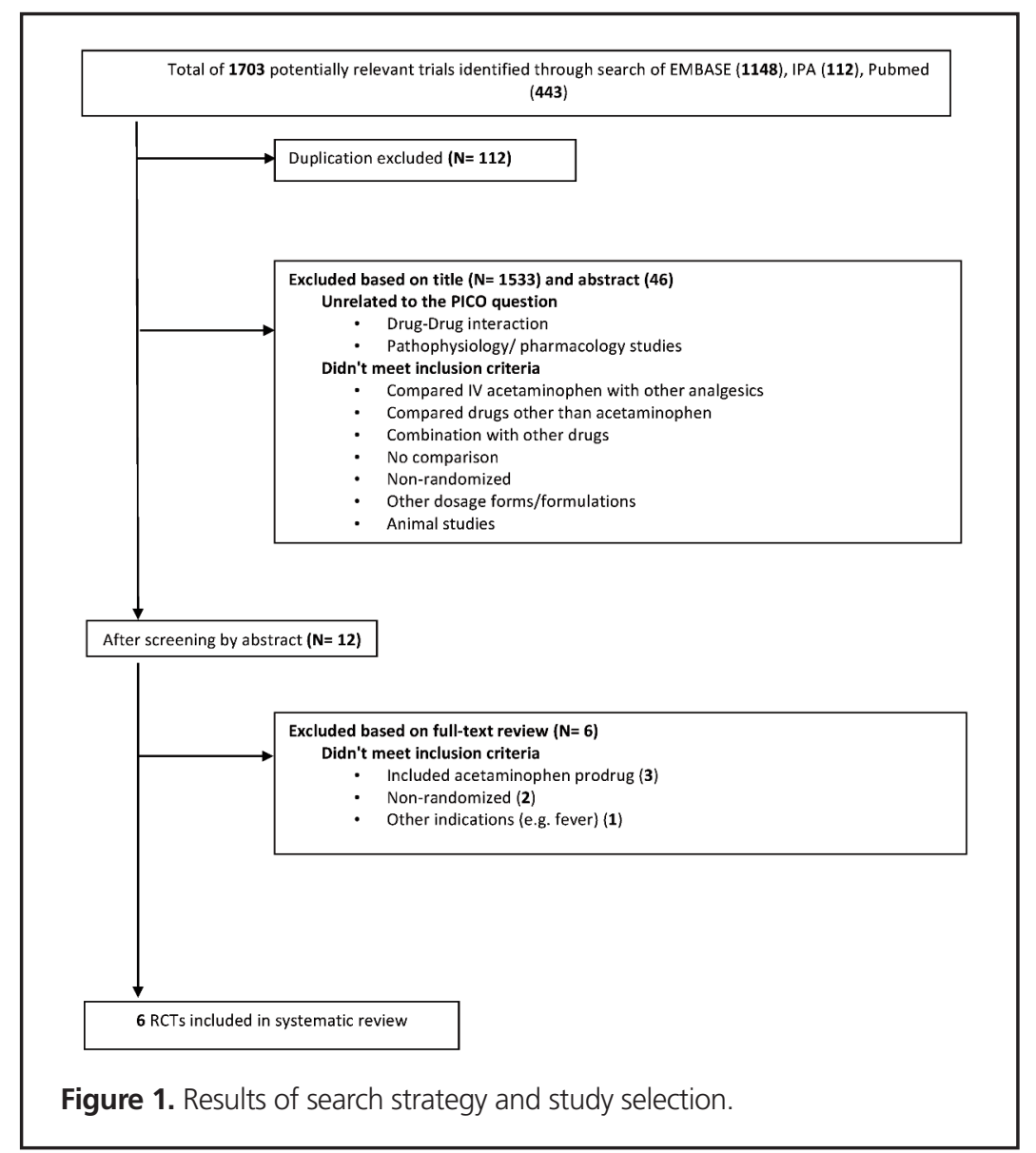


Table 1. Summary of Study Characteristics (part 1 of 2)

\begin{tabular}{|c|c|c|c|c|c|}
\hline Study & Objective(s) & Design & Participants & Interventions & Outcomes \\
\hline $\begin{array}{l}\text { Ameer et al. } \\
(1983)^{21}\end{array}$ & $\begin{array}{l}\text { To determine } \\
\text { absolute } \\
\text { bioavailability } \\
\text { of acetaminophen } \\
\text { in tablet and elixir } \\
\text { dosage forms }\end{array}$ & $\begin{array}{l}\text { 3-way prospective } \\
\text { randomized crossover } \\
\text { study of absolute } \\
\text { bioavailability in } \\
\text { healthy volunteers }\end{array}$ & $\begin{array}{l}18 \text { healthy volunteers } \\
\text { ( } 8 \text { women), } \\
\text { age } 22-36 \text { yr, with } \\
\text { no history of chronic } \\
\text { diseases and not } \\
\text { concurrently taking } \\
\text { other medications }\end{array}$ & $\begin{array}{l}\text { Participants received } \\
\text { three } 650 \text {-mg doses } \\
\text { of acetaminophen } \\
\text { as } 5 \text {-min IV bolus, } \\
\text { two } 325 \text {-mg tablets, } \\
\text { or } 19.5 \mathrm{~mL} \text { elixir on } \\
3 \text { separate phases, } \\
\text { with } 1 \text {-wk washout } \\
\text { period between } \\
\text { phases } \\
\text { Oral doses preceded } \\
\text { by overnight fasting } \\
\text { and followed by } 3 \mathrm{~h} \\
\text { fasting } \\
\text { Serial blood samples } \\
\text { collected up to } 12 \mathrm{~h} \\
\text { after each dose }\end{array}$ & $\begin{array}{l}\text { Acetaminophen } \\
\text { volume of distribution, } \\
\text { half-life, clearance, } \\
C_{\max } T_{\max } \text { lag time, } \\
\text { AUC }_{0-\infty} \text { and absolute } \\
\text { bioavailability }\end{array}$ \\
\hline $\begin{array}{l}\text { Pettersson et al. } \\
(2005) \star 22\end{array}$ & $\begin{array}{l}\text { To compare opioid } \\
\text { consumption and } \\
\text { opioid-related adverse } \\
\text { effects in ICU patients } \\
\text { receiving IV or oral } \\
\text { acetaminophen }\end{array}$ & $\begin{array}{l}\text { Prospective, } \\
\text { randomized, open- } \\
\text { label, parallel-group } \\
\text { study in ICU patients } \\
\text { undergoing elective } \\
\text { coronary artery bypass } \\
\text { graft with } \\
\text { cardiopulmonary } \\
\text { bypass }\end{array}$ & $\begin{array}{l}80 \text { patients } \\
\text { ( } 3 \text { excluded for } \\
\text { incomplete data) } \\
\text { randomized into } \\
2 \text { groups: } \\
\text { PO group }(n=38) \text { : } \\
32 \text { men, } \\
\text { age } 66 \pm 9 \mathrm{yr}, \\
\text { weight } 81 \pm 16 \mathrm{~kg} \\
\text { IV group ( } n=39) \text { : } \\
32 \text { men, age } \\
66 \pm 9 \mathrm{yr} \text {, weight } \\
79 \pm 14 \mathrm{~kg}\end{array}$ & $\begin{array}{l}\text { PO group: } 1 \mathrm{~g} \text { oral } \\
\text { acetaminophen q6h } \\
\text { after surgery } \\
\text { IV group: } 1 \mathrm{~g} \text { IV } \\
\text { acetaminophen q6h } \\
\text { after surgery } \\
\text { Nausea and vomiting } \\
\text { treated with } \\
\text { ondansetron } \\
\text { Premedication with } \\
\text { morphine or } \\
\text { ketobemidone }\end{array}$ & $\begin{array}{l}\text { Opioid requirements } \\
\text { (amount of opioid } \\
\text { administered during } \\
\text { study period) } \\
\text { Pain level, using } \\
100-m m \text { VAS } \\
\text { Core temperature } \\
\text { Incidence of } \\
\text { postoperative nausea } \\
\text { and vomiting }\end{array}$ \\
\hline $\begin{array}{l}\text { Van der } \\
\text { Westhuizen et al. } \\
(2011)+\dagger^{23}\end{array}$ & $\begin{array}{l}\text { To compare } \\
\text { acetaminophen } \\
\text { concentrations in } \\
\text { plasma following } \\
\text { administration of a } \\
\text { single preoperative } \\
\text { dose of oral or IV } \\
\text { acetaminophen }\end{array}$ & $\begin{array}{l}\text { Prospective, } \\
\text { randomized, open- } \\
\text { label, parallel-group } \\
\text { study in patients } \\
\text { scheduled for elective } \\
\text { ear, nose, and throat } \\
\text { surgery or orthopedic } \\
\text { surgery }\end{array}$ & $\begin{array}{l}113 \text { patients (7 } \\
\text { excluded) with ASA } \\
\text { physical status I or II } \\
\text { (normal healthy - } \\
\text { mild systemic disease) } \\
\text { randomized into } 2 \\
\text { groups: } \\
\text { PO group ( } n=52) \text { : } \\
22 \text { women, age } \\
46.3 \text { yr }(16-74 \text { yr), } \\
\text { weight } 83.2 \mathrm{~kg} \\
\text { (49-129 yr) } \\
\text { IV group ( } n=54) \text { : } \\
22 \text { women, age } \\
43.6 \text { yr (39.3-48 yr), } \\
\text { weight } 81.4 \mathrm{~kg} \\
\text { (77.1-85.7 kg) } \\
\text { Patients excluded if } \\
\text { already taking } \\
\text { acetaminophen, } \\
\text { had pancreatic disease } \\
\text { in last } 12 \text { months, } \\
\text { or had hepatic or } \\
\text { renal impairment }\end{array}$ & $\begin{array}{l}\text { PO group: } 1 \mathrm{~g} \text { oral } \\
\text { acetaminophen } \\
30 \text { min preoperatively } \\
\text { IV group: } 1 \mathrm{~g} \mathrm{IV} \\
\text { acetaminophen } \\
\text { immediately before } \\
\text { induction of } \\
\text { anesthesia } \\
\text { Serial blood samples } \\
\text { collected up to } 240 \\
\text { min after each dose } \\
\text { No acetaminophen } \\
\text { allowed } 12 \text { h before } \\
\text { study }\end{array}$ & $\begin{array}{l}\text { Postoperative plasma } \\
\text { acetaminophen } \\
\text { concentrations }\end{array}$ \\
\hline
\end{tabular}


This single copy is for your personal, non-commercial use only.

For permission to reprint multiple copies or to order presentation-ready copies for distribution, contact CJHP at cjhpedit@cshp.ca

Table 1. Summary of Study Characteristics (part 2 of 2)

\begin{tabular}{|c|c|c|c|c|c|}
\hline Study & Objective(s) & Design & Participants & Interventions & Outcomes \\
\hline $\begin{array}{l}\text { Brett et al. } \\
(2012) \ddagger^{4}\end{array}$ & $\begin{array}{l}\text { To compare } \\
\text { acetaminophen } \\
\text { concentrations in } \\
\text { plasma in early } \\
\text { postoperative phase } \\
\text { following } \\
\text { intraoperative IV and } \\
\text { preoperative oral } \\
\text { acetaminophen }\end{array}$ & $\begin{array}{l}\text { Prospective, } \\
\text { randomized, double- } \\
\text { blind, parallel-group } \\
\text { study in patients } \\
\text { scheduled for day- } \\
\text { case knee arthroscopy } \\
\text { under genera } \\
\text { anesthesia }\end{array}$ & $\begin{array}{l}30 \text { patients with } \\
\text { ASA physical status } \\
\text { I-I (normal healthy - } \\
\text { mild systemic disease) } \\
\text { randomized into } \\
2 \text { groups: } \\
\text { PO group }(n=20) \text { : } \\
8 \text { women, age } \\
50.3 \pm 14.7 \mathrm{yr}, \\
\text { BMl } 27.9 \pm 4.1 \mathrm{~kg} / \mathrm{m}^{2} \\
\text { IV group }(n=10): \\
3 \text { women, age } \\
50.7 \pm 13.3 \mathrm{yr}, \\
\text { BMl } 27.5 \pm 4.4 \mathrm{~kg} / \mathrm{m}^{2}\end{array}$ & $\begin{array}{l}\text { PO group: } 1 \mathrm{~g} \text { oral } \\
\text { acetaminophen } \\
\text { 30-60 min } \\
\text { preoperatively } \\
\text { IV group: } 1 \mathrm{~g} \\
\text { acetaminophen IV } \\
\text { infusion over } 15 \mathrm{~min} \\
\text { Intraoperatively and } \\
\text { oral placebo } \\
\text { preoperatively to } \\
\text { ensure blinding } \\
\text { IV fentanyl used in } \\
\text { both groups for } \\
\text { intraoperative } \\
\text { analgesia and as } \\
\text { postoperative rescue } \\
\text { analgesic if VAS } \\
\text { score }>30 \text { mm }\end{array}$ & $\begin{array}{l}\text { Plasma concentration } \\
\text { of acetaminophen } \\
30 \text { min after surgery } \\
\text { (primary) } \\
\text { Postoperative VAS } \\
\text { scores (100-mm scale } \\
\text { assessed at 10-min } \\
\text { intervals while patient } \\
\text { awake in recovery } \\
\text { room) } \\
\text { Postoperative fentanyl } \\
\text { requirements } \\
\text { Length of stay in } \\
\text { recovery area }\end{array}$ \\
\hline $\begin{array}{l}\text { Singla et al. } \\
(2012)^{25}\end{array}$ & $\begin{array}{l}\text { To compare plasma } \\
\text { and CSF } \\
\text { concentration-time } \\
\text { curves and PK } \\
\text { parameters for } \\
\text { acetaminophen after } \\
\text { IV, oral, and rectal } \\
\text { administration }\end{array}$ & $\begin{array}{l}\text { Prospective, } \\
\text { randomized, open- } \\
\text { label 3-way } \\
\text { crossover study }\end{array}$ & $\begin{array}{l}7 \text { healthy nonsmoking } \\
\text { men, age } 19-44 \mathrm{yr}, \\
\text { BMl } 19-25.6 \mathrm{~kg} / \mathrm{m}^{2} \text {, } \\
\text { weight } \geq 50 \mathrm{~kg} \\
\text { No medications for } \\
7 \text { days before study; } \\
\text { no history of excessive } \\
\text { bleeding, recent } \\
\text { infection, elevated } \\
\text { intracranial pressure, } \\
\text { neurological disease, } \\
\text { or lumbar spine } \\
\text { deformities }\end{array}$ & $\begin{array}{l}\text { Participants received } \\
1-g \text { dose of } \\
\text { acetaminophen IV } \\
\text { infusion over } 15 \text { min, } \\
1-g \text { dose of oral } \\
\text { acetaminophen, and } \\
1.3-g \text { dose of } \\
\text { acetaminophen rectal } \\
\text { suppositories, with } \\
\text { 24-h washout period } \\
\text { between doses } \\
\text { Serial plasma and CSF } \\
\text { samples collected up } \\
\text { to } 6 \mathrm{~h} \text { after each dose }\end{array}$ & $\begin{array}{l}\text { Acetaminophen } C_{\max } \\
T_{\max } \text { and AUC } \mathrm{C}_{0-6} \text { in } \\
\text { plasma and CSF } \\
\text { Plasma half-life of } \\
\text { acetaminophen } \\
\text { Regular safety } \\
\text { assessments and } \\
\text { self-reporting of } \\
\text { adverse effects }\end{array}$ \\
\hline $\begin{array}{l}\text { Fenlon et al. } \\
(2013)^{26}\end{array}$ & $\begin{array}{l}\text { To compare } \\
\text { postoperative analgesia } \\
\text { after preoperative } \\
\text { oral and IV } \\
\text { acetaminophen }\end{array}$ & $\begin{array}{l}\text { Prospective, } \\
\text { randomized, double- } \\
\text { blind, double-dummy } \\
\text { parallel-group non- } \\
\text { inferiority study in } \\
\text { patients visiting a } \\
\text { maxillofacial } \\
\text { outpatient clinic }\end{array}$ & $\begin{array}{l}128 \text { patients, age } \\
18-65 \mathrm{yr}, \text { who } \\
\text { underwent at least } \\
\text { one lower third molar } \\
\text { extraction under } \\
\text { general anesthesia } \\
\text { randomized into } \\
2 \text { groups: PO group } \\
\text { ( } n=65): 51 \text { women, } \\
\text { age } 18.1-57.7 \mathrm{yr}, \\
\text { BMl } 24.4 \pm 4.3 \mathrm{~kg} / \mathrm{m}^{2} \\
\text { IV group }(n=63): \\
43 \text { women, age } \\
18.7-54.4 \mathrm{yr}, \\
\text { BMl } 24.5 \pm 5.0 \mathrm{~kg} / \mathrm{m}^{2} \\
\text { No analgesics or } \\
\text { caffeine for } 24 \mathrm{~h} \text { and } \\
6 \mathrm{~h} \text { before study, } \\
\text { respectively }\end{array}$ & $\begin{array}{l}\text { PO group: } 1 \mathrm{~g} \text { oral } \\
\text { acetaminophen at } \\
\text { least } 45 \text { min before } \\
\text { surgery } \\
\text { IV group: } 1 \mathrm{~g} \mathrm{IV} \\
\text { acetaminophen } \\
\text { immediately after } \\
\text { induction of } \\
\text { anesthesia } \\
\text { Both groups received } \\
\text { appropriate placebo } \\
\text { (oral tablets or } \\
\text { IV } 0.9 \% \text { normal saline) } \\
\end{array}$ & $\begin{array}{l}100-m m \text { VAS score at } \\
1 \mathrm{~h} \text { after surgery } \\
\text { Time to request rescue } \\
\text { analgesia } \\
\text { VAS score at time of } \\
\text { rescue analgesia } \\
\text { Any adverse events } \\
\text { reported }\end{array}$ \\
\hline
\end{tabular}

$\overline{\mathrm{ASA}}=$ American Society of Anesthesiologists, $\mathrm{AUC}_{0-\infty}=$ area under the plasma concentration curve, $\mathrm{AUC}_{0-6}=$ area under the plasma concentration-time curve from 0 to $6 \mathrm{~h}, \mathrm{BMl}=$ body mass index, $C_{\max }=$ maximum concentration, $C \mathrm{CSF}=$ cerebrospinal fluid, ICU = intensive care unit, IV = intravenous, $\mathrm{PK}=$ pharmacokinetic, $\mathrm{PO}=$ oral, $\mathrm{q} 6 \mathrm{~h}=$ every $6 \mathrm{~h}, T_{\max }=$ time to maximum concentration, VAS = visual analogue scale.

*Baseline characteristics presented as mean \pm standard deviation.

†Baseline characteristics presented as mean (range).

$\ddagger$ Baseline characteristics presented as mean \pm standard error. 
This single copy is for your personal, non-commercial use only.

For permission to reprint multiple copies or to order presentation-ready copies for distribution, contact CJHP at cjhpedit@cshp.ca

Table 2. Summary of Clinical Efficacy, Safety, and Pharmacokinetic Results

Study Clinical Efficacy

Ameer et al. (1983)*21 Not evaluated
Clinical Safety

Not evaluated
Pharmacokinetic

Tmax was significantly shorter with elixir

than with tablet $(0.48 \pm 0.06 \mathrm{~h}$ vs.

$0.76 \pm 0.12 \mathrm{~h} ; p<0.025$ )

Absolute bioavailability was significantly

higher with elixir than with tablet

$(87 \% \pm 2 \%$ vs. $79 \% \pm 2 \% ; p<0.001)$

No statistically significant differences between elixir and tablet preparations in any other PK parameter

\begin{aligned} \hline Pettersson et al. (2005)+22 & Amount of opioid used \\ & postoperatively was significantly \\ & ower with IV than with oral \\ & acetaminophen $(1.5 \pm 0.5 \mathrm{mg} / \mathrm{kg} \\ &$ vs. $1.9 \pm 0.6 \mathrm{mg} / \mathrm{kg} ; \mathrm{p}=0.008) \\ &$ No statistically significant differences \\ & in incidence of postoperative nausea \\ & and vomiting, increase in body \\ & temperature, or mean VAS scores \\ & during ICU stay \end{aligned}

Van der Westhuizen Not evaluated

Not evaluated

Not evaluated et al. $(2011)^{23}$

Not evaluated
No treatment-related side effects observed in either group
Therapeutic acetaminophen concentrations in plasma (> $10 \mathrm{mg} / \mathrm{L}$ ) were achieved at any time in $96 \%$ of patients in IV group vs. $67 \%$ of patients in PO group $(p<0.0001)$ Median Cmax was significantly higher for IV group than for PO group $19 \mathrm{mg} / \mathrm{L}$ [IQR 15-23] vs. $13 \mathrm{mg} / \mathrm{L}$ [IQR <10-18]; $p<0.0001)$

Therapeutic acetaminophen concentrations maintained for $3 \mathrm{~h}$ with IV vs. $1.5 \mathrm{~h}$ with PO

\begin{tabular}{ll}
\hline Brett et al. (2012)*24 & Mean pain score at 50 min after \\
& surgery was significantly higher \\
& with PO than with IV administration \\
& $(30.8 \pm 5.8 \mathrm{~mm}$ vs. $11.6 \pm 2.8 \mathrm{~mm} ;$ \\
& $p=0.025)$ \\
& No statistically significant differences \\
& in fentanyl requirements, mean pain \\
& score at 30 min, or total time in \\
& recovery area
\end{tabular}

Singla et al. (2012)+25 Not evaluated

\section{No treatment-related side effects observed in} either group (n)

Three participants

Mean acetaminophen concentrations 30 min after surgery were significantly higher with IV than with oral administration $(88.6 \pm 16.3 \mu \mathrm{mol} / \mathrm{L}$ vs. $53.2 \pm 19.1 \mu \mathrm{mol} / \mathrm{L}$; $p=0.0005)$

All patients in IV group had plasma concentrations above proposed analgesic level $(66 \mu \mathrm{mol} / \mathrm{L})$ vs. only $35 \%$ of patients in $\mathrm{PO}$ group

Mean plasma $C_{\max }$ was significantly higher experienced a total of 12 adverse reactions (e.g., postdural puncture headache, nausea, viral infection); none were deemed to be treatment-related

Three participants
experienced a total of
12 adverse reactions
(e.g., postdural puncture
headache, nausea, viral
infection); none were
deemed to be
treatment-related
with IV than with PO administration $(21.6 \pm 3.9 \mu \mathrm{g} / \mathrm{mL}$ vs. $12.3 \pm 5.6 \mu \mathrm{g} / \mathrm{mL}$; $p=0.0004)$ and $T_{\max }$ was significantly shorter $(0.25 \mathrm{~h}$ vs. $1 \mathrm{~h} ; p=0.0018)$ Mean $A_{U} C_{0-6} 45 \%$ higher with IV than with PO administration ( $p$ value not reported)

No statistically significant difference in plasma half-life between the 2 groups Mean CSFmax was significantly higher with IV than with $\mathrm{PO}$ administration $(5.94 \pm 1.09 \mu \mathrm{g} / \mathrm{mL}$ vs. $3.72 \pm 1.45 \mu \mathrm{g} / \mathrm{mL}$; $p<0.0001)$ with no statistically significant differences in $T_{\max }$ Mean CSF $A \cup C_{0-6}$ was significantly higher with IV than with PO administration $(24.9 \pm 4.3 \mu \mathrm{g} \cdot \mathrm{h} / \mathrm{mL}$ vs. $14.2 \pm 7.4$ $\mu \mathrm{g} \cdot \mathrm{h} / \mathrm{mL} ; p=0.009$ )

\begin{tabular}{|c|c|c|}
\hline Fenlon et al. $(2013)^{26}$ & $\begin{array}{l}\text { Proportion of patients with VAS } \\
\leq 30 \mathrm{~mm} \text { at } 1 \text { h postoperatively } \\
\text { showed non-inferiority of PO to IV } \\
\text { acetaminophen }(23.1 \% \\
{[95 \% \mathrm{Cl} 14 \%-35 \%] \mathrm{Vs} .27 .0 \%} \\
[95 \% \mathrm{CI} 17 \%-40 \%]) \\
\text { No statistically significant differences } \\
\text { in mean VAS scores, requirements } \\
\text { for rescue analgesia, or time to } \\
\text { rescue analgesia }\end{array}$ & $\begin{array}{l}\text { Dizziness after surgery } \\
(n=1) \text { and burn to the } \\
\text { lip from surgical drill } \\
(n=1) \text {; both deemed } \\
\text { unrelated to } \\
\text { acetaminophen } \\
\text { treatment }\end{array}$ \\
\hline
\end{tabular}

$\mathrm{AUC}_{0-6}=$ area under the plasma concentration time curve from 0 to $6 \mathrm{~h}, \mathrm{Cl}=$ confidence interval, $C_{\max }=$ maximum concentration, $\mathrm{CSF}=$ cerebral spinal fluid, CSFmax = maximum concentration in cerebrospinal fluid, ICU = intensive care unit, IQR = interquartile range, IV = intravenous, PK = pharmacokinetic, PO = oral, $T_{\max }=$ time to maximum concentration, VAS = visual analogue scale. *Results presented as mean \pm standard error.

†Results presented as mean \pm standard deviation. 
This single copy is for your personal, non-commercial use only.

For permission to reprint multiple copies or to order presentation-ready copies for distribution, contact CJHP at cjhpedit@cshp.ca

Table 3. Assessment of Risk of Bias

Domain; Risk of Bias*

\begin{tabular}{|c|c|c|c|c|c|c|}
\hline Study & $\begin{array}{l}\text { Sequence } \\
\text { Generation }\end{array}$ & $\begin{array}{l}\text { Allocation } \\
\text { Concealment }\end{array}$ & Blinding & $\begin{array}{c}\text { Incomplete } \\
\text { Data Outcome }\end{array}$ & $\begin{array}{c}\text { Selective Outcome } \\
\text { Reporting }\end{array}$ & Other \\
\hline $\begin{array}{l}\text { Ameer et al. } \\
(1983) \dagger^{21}\end{array}$ & Unclear & Unclear & Low & Low & Low & Unclear \\
\hline $\begin{array}{l}\text { Pettersson et al. } \\
(2005)^{22}\end{array}$ & Unclear & Unclear & High & Unclear & Low & Low \\
\hline $\begin{array}{l}\text { Van der } \\
\text { Westhuizen } \\
\text { et al. }(2001) \dagger^{23}\end{array}$ & Low & Unclear & Low & Low & Unclear & Low \\
\hline $\begin{array}{l}\text { Brett et al. } \\
(2012)^{24}\end{array}$ & Low & Unclear & Unclear & Low & Low & Low \\
\hline $\begin{array}{l}\text { Singla et al. } \\
(2012)^{25}\end{array}$ & Unclear & Unclear & Low & Low & Unclear & Unclear \\
\hline $\begin{array}{l}\text { Fenlon et al. } \\
(2013)^{26}\end{array}$ & Low & Low & Low & Low & Low & Unclear \\
\hline
\end{tabular}

*Risk of bias was categorized as low, high, or unclear.

tOpen-label study with low risk of bias because of objective pharmacokinetic outcomes.

receiving acetaminophen by the IV route than in the group receiving acetaminophen by the oral route (mean \pm standard deviation $17.4 \pm 7.9 \mathrm{mg}$ versus $22.1 \pm 8.6 \mathrm{mg} ; p<0.05$ ). However, this difference did not translate into a significant difference in rates of postoperative nausea and vomiting or any significant difference in pain scores on a $100-\mathrm{mm}$ visual analogue scale (VAS) at any time. Brett and others ${ }^{24}$ compared postoperative pain scores, length of stay in recovery, and opioid-sparing effects of oral and IV acetaminophen in patients who had undergone knee arthroscopy. No significant differences were found in terms of fentanyl requirements or length of stay in recovery. The only significant difference was in VAS scores (on a 100-mm scale), in favour of IV over oral administration (mean \pm standard error $11.6 \pm 2.8 \mathrm{~mm}$ versus $30.8 \pm 5.8 \mathrm{~mm} ; p=0.025$ ) at $50 \mathrm{~min}$ after arrival in recovery. However, it was noted that one-third of the patients had been discharged by this time point. Fenlon and others $^{26}$ assessed VAS scores (on a 100-mm scale) as well as requests for and timing of rescue analgesia in patients undergoing extraction of the third molar. A non-inferiority margin was set at $20 \%$ difference in satisfactory pain control, defined as VAS scores $\leq 30 \mathrm{~mm}$ at $1 \mathrm{~h}$ postoperatively on a 100-mm VAS. This threshold was achieved in $23.1 \%$ of patients in the oral administration group and $27.0 \%$ of those in the IV administration group, giving a difference in proportions of $-0.039(90 \%$ CI -0.17 to 0.09 ). Because this result was within the $20 \%$ non-inferiority margin, oral acetaminophen was deemed noninferior to IV acetaminophen. However, concerns about the study design and the statistical analysis were noted and are discussed below. No statistically significant differences were found in terms of requests for and timing of rescue analgesia.

\section{Studies Reporting Safety Outcomes}

Four of the studies reported on adverse events, ${ }^{23-26}$ but only 2 of these studies reported the occurrence of any adverse event. ${ }^{25,26}$ None of the adverse events was deemed to be associated with acetaminophen use.

\section{Studies Reporting Pharmacokinetic Outcomes}

Four of the studies reported pharmacokinetic outcomes. ${ }^{21,23-25}$ Ameer and others ${ }^{21}$ found that single doses of acetaminophen tablets or elixir achieved high bioavailability relative to IV formulations. The elixir formulation had significantly higher bioavailability than the tablets $(87 \%$ versus $79 \% ; p<0.001)$ and shorter $T_{\max }(0.48 \mathrm{~h}$ versus $0.76 \mathrm{~h} ; p<0.025)$. Singla and others $^{25}$ reported $75 \%$ and $45 \%$ higher plasma $C_{\max }$ and $\mathrm{AUC}_{0-6}$, respectively, following IV dosing relative to oral dosing. Furthermore, peak concentrations and $\mathrm{AUC}_{0-6}$ in cerebrospinal fluid were $60 \%$ higher and $75 \%$ higher, respectively, after IV dosing relative to oral dosing. Van der Westhuizen and others ${ }^{23}$ found that $96 \%$ of patients receiving the IV formulation achieved a target plasma acetaminophen concentration above $10 \mathrm{mg} / \mathrm{L}$, as compared with only $67 \%$ in the oral group. Those results were confirmed by a significantly higher median $C_{\text {max }}$ after IV dosing relative to oral dosing $(19 \mathrm{mg} / \mathrm{L}$ versus 13 $\mathrm{mg} / \mathrm{L} ; p<0.0001)$. Similar results were reported by Brett and others, ${ }^{24}$ with $100 \%$ of patients in the IV group achieving plasma concentrations above $10 \mathrm{mg} / \mathrm{L}$, compared with only $35 \%$ in the oral group. There was also a significant difference in mean plasma acetaminophen concentrations at $30 \mathrm{~min}$ after arrival in the recovery room in favour of the IV group $(88.6 \mu \mathrm{mol} / \mathrm{L}$ versus 53.2 $\mu \mathrm{mol} / \mathrm{L} ; p=0.0005)$.

\section{Assessment of Risk of Bias}

The risk-of-bias assessments are summarized in Table 3. Notably, studies that assessed only pharmacokinetic outcomes were generally deemed to have low risks of performance and detection biases (blinding domain), even if they were not blinded. 
In most cases, sequence generation was not reported in enough detail to assess randomization methods. Only one study reported allocation concealment methods. ${ }^{26}$ The study by Pettersson and others ${ }^{22}$ was the only one to receive a "high risk of bias" rating for the blinding domain, because of the open-label design and inclusion of outcomes that were subjective in nature. That study was also deemed to have an "unclear risk of bias" for the incomplete outcome data domain, because of the unjustified exclusion of data for 3 patients from the final data analysis. Studies were assigned a judgment of "unclear risk" of reporting bias if the study protocol was not available, and outcomes of interest were not prespecified in the methods section, for comparison with reported outcomes. ${ }^{23,25}$ Other examples of unclear bias included lack of sample size calculation ${ }^{21,25}$ and imbalance in patients' baseline characteristics. ${ }^{26}$ Fenlon and others ${ }^{26}$ used a 90\% CI to assess non-inferiority between dosage forms. This practice narrows the CI and may bias the results in favour of achieving non-inferiority. Other biases relating to study design were identified and deemed unclear (see Table 3).

\section{DISCUSSION}

This review has summarized and evaluated the available published literature describing efficacy, safety, and pharmacokinetic outcomes of randomized studies assessing oral versus IV dosage forms of acetaminophen. Within studies conducted in populations requiring analgesia, the indications for pain control were diverse and inconsistent between studies. Of the 6 identified studies, 3 reported on efficacy, 4 on safety, and 4 on pharmacokinetic outcomes. The findings of each study were largely hypothesis-generating and can provide insight for the design of future randomized controlled trials attempting to assess these outcomes.

A major finding of this review was the absence of strong evidence suggesting superiority of IV acetaminophen administration over oral routes. Significant end points in the Pettersson and Brett trials for efficacy are called into question on the basis of clinical relevance and study design. ${ }^{22,24}$ For example, the significantly lower use of rescue opioids (ketobemidone) in the IV group in the study by Pettersson and others ${ }^{22}$ (17.4 mg versus $22.1 \mathrm{mg}$ ) is not clinically significant and would not justify preferentially giving postoperative IV therapy to patients who have undergone coronary artery bypass grafting. Similarly, the significant finding in the trial by Brett and others ${ }^{24}$ of a lower VAS score in the IV group at $50 \mathrm{~min}$ after entrance to recovery must be questioned, since one-third of patients had been discharged by that point. Therefore, on the basis of current evidence, if a patient has a functioning gastrointestinal tract and is able to take oral formulations, IV formulations are not indicated.

A systematic review published by the Cochrane Collaboration provides insight into the effect of IV acetaminophen in comparison with opioids and other nonsteroidal anti-inflammatory drugs. ${ }^{27}$
Although no differences were found between groups of identified studies in terms of pain reduction, the results must be interpreted cautiously, as small sample sizes precluded testing for superiority. It was noted, however, that IV acetaminophen may be useful for opioid-sparing in postoperative pain. ${ }^{27}$ To date, no strong evidence exists that IV acetaminophen should replace any form of standard care. At most, the evidence indicates that this formulation could function as an adjunctive agent in patients unable to take oral forms.

Among the studies reporting safety outcomes, none reported this information in enough detail to allow critical evaluation and formulation of conclusions. Although no treatment-related adverse effects were noted in any study, IV routes of administration are inherently associated with higher rates of adverse effects, as mentioned earlier. Additionally, all studies were based on single doses or short-term therapy, and the risks of long-term IV dosing could not be documented.

Four of the 6 studies evaluated pharmacokinetic outcomes, with only one study combining both pharmacokinetic and clinical efficacy outcomes. ${ }^{24}$ Ameer and others ${ }^{21}$ found that oral dosage forms of acetaminophen were associated with high bioavailability, with only $13 \%$ to $21 \%$ of the dose being lost during absorption. Similar results were obtained previously by Rawlins and others, ${ }^{28}$ who showed that bioavailability of acetaminophen tablet dosage forms increased from 63\% after 500-mg doses to $89 \%$ after 1000-mg doses. Such bioavailability at doses used in the clinical setting may indicate dose equivalency between IV and oral dosage forms, which enhances interchangeability in the absence of efficacy differences.

Most studies including pharmacokinetic outcomes reported higher $C_{\max }$, shorter $T_{\max }$, higher postoperative plasma concentrations, and larger proportions of patients achieving target plasma concentrations after IV dosing compared with oral dosing. Such results may indicate superior efficacy for IV dosing; however, this can be confirmed only through a well-established concentration-response relationship, which is currently not available for acetaminophen. Target concentrations used in studies by Brett and others ${ }^{24}$ and van der Westhuizen and others ${ }^{23}$ are only proposed targets and have not been consistently shown to relate to analgesic outcomes in previous literature. Furthermore, Brett and others ${ }^{24}$ combined pharmacokinetic with clinical efficacy outcomes in an attempt to establish such a relationship. However, although their results showed higher plasma concentrations of acetaminophen after IV dosing than after oral dosing, this difference did not translate into differences in clinical efficacy. On the contrary, fentanyl requirements and mean VAS scores at the time of measurement of plasma concentrations were similar between the 2 groups. Such results indicate that differences in plasma exposure between IV and oral dosing may not necessarily result in differences in clinical outcomes.

At the time of writing (late 2014), IV acetaminophen was not widely available in Canada, yet was in common use in the 
United States, Europe, the Middle East, and Asia. In the United States, there has been great debate regarding use of this formulation, which has led many hospitals to adopt policies and procedures that restrict use for a limited period or for patients not able to take medications by mouth. These restrictions are required because of the cost of the product, in addition to other administration-related inconveniences. ${ }^{19}$ Canadian hospitals and formulary committees should be aware of the available efficacy and safety data if the formulation is marketed in Canada and its use becomes widespread. Given the high cost and the lack of superiority over oral forms, Canadian hospitals may need to restrict use of the IV formulation, as their US counterparts have already done. However, these decisions will depend on marketing of the product in Canada. In addition to institution-based decision-making, pharmacists and other health care professionals should have baseline knowledge about this comparison in the event of drug information requests or for the education of patients or physicians coming from other regions where access has already been approved.

Recommendations can be made regarding future research in this area. According to anecdotal information, specific patient populations of interest are those with advanced cancer pain or trauma-related injuries, as well as those undergoing or recovering from surgical procedures. It is within these populations that IV therapy is commonly advocated, even when oral therapy can be tolerated. Studies should include both efficacy and safety outcomes and should assess multiple dosing (rather than single dosing) to better characterize longer-term use and differences relating to the dosage forms used in practice. Such studies could also include pharmacokinetic outcomes to allow a better understanding of pharmacokinetic-pharmacodynamic relationships and how parameters such as bioavailability, onset of action, and first-pass metabolism influence both efficacy and safety.

This study had some limitations. First, the studies identified were diverse in population, dosage regimens, and reported outcomes. This diversity inhibited the pooling of data by quantitative methods. Also, the identified studies were limited in number, which precluded generalizing results to all patients for whom acetaminophen therapy is indicated. Lastly, study quality markers were not consistently reported, which made the risk-of-bias assessment unclear for a large number of categories.

Despite these limitations, the data presented in this review allow for certain conclusions to be drawn. It is evident from the studies identified that there is no clear indication for IV acetaminophen over oral acetaminophen if patients have a functioning gastrointestinal tract and are able to take oral dosage forms. Although safety data were largely not assessed, decisionmaking must consider the known risks associated with IV administration before this form of administration is commonly recommended for patients who are able to take oral therapy. This situation represents an opportunity for pharmacists to be patient advocates by ensuring that clinicians are not inappropriately advocating IV therapy in practice. If the IV formulation is eventually approved in Canada, restriction policies and procedures may need to be established to prevent increases in health care costs and labour requirements without any major benefits in terms of efficacy and safety. Finally, future studies should focus on assessing multiple-dose regimens over longer periods for patients with common pain indications such as cancer, trauma, and surgery.

\section{References}

1. Kaufman DW, Kelly JP, Rosenberg L, Anderson TE, Mitchell AA. Recent patterns of medication use in the ambulatory adult population of the United States: the Slone survey. JAMA. 2002;287(3):337-44.

2. Duggan ST, Scott LJ. Intravenous paracetamol (acetaminophen). Drugs. 2009;69(1):101-13.

3. Prescott LF. The therapeutic use of paracetamol. In: Paracetamol (acetaminophen): a critical bibliographic review. London (UK): Taylor and Francis; 1996. p. 253-99.

4. Breivik H. Postoperative pain: towards optimal pharmacological and epidural analgesia. In: Giamberardino MA, editor. Pain 2002 — an updated review: refresher course syllabus. Seattle (WA): IASP Press; 2002. p. 337-49.

5. Prescott LF. Paracetamol: past, present, and future. Am J Ther. 2000; 7(2):143-7.

6. Candiotti KA, Bergese SD, Viscusi ER, Singla SK, Royal MA, Singla NK. Safety of multiple-dose intravenous acetaminophen in adult inpatients. Pain Med. 2010;11(12):1841-8.

7. Van Aken H, Thys L, Veekman L, Buerkle H. Assessing analgesia in single and repeated administrations of propacetamol for postoperative pain: comparison with morphine after dental surgery. Anesth Analg. 2004; 98(1):159-65.

8. Waknine Y. FDA approves first intravenous formulation of acetaminophen. In: Medscape Medical News. New York (NY): WebMD LLC; 2010 [cited 2014 Nov 21]. Available from: www.medscape.com/viewarticle/731994. Subscription required to access content.

9. Malaise O, Bruyere O, Reginster JY. Intravenous paracetamol: a review of efficacy and safety in therapeutic use. Future Neurol. 2007;2(6):673-88.

10. Apfel CC, Souza K, Portillo J, Dalal P, Bergese SD. Patient satisfaction with intravenous acetaminophen: a pooled analysis of five randomized, placebocontrolled studies in the acute postoperative setting. J Healthc Qual. 2014 Jan 16. doi: 10.1111/jhq.12062. [Epub ahead of print].

11. Arana A, Morton NS, Hansen TG. Treatment with paracetamol in infants. Acta Anaesthesiol Scand. 2001;45(1):20-9.

12. Moller PL, Sindet-Pedersen S, Petersen CT, Juhl GI, Dillenschneider A, Skoglund LA. Onset of acetaminophen analgesia: comparison of oral and intravenous routes after third molar surgery. Br J Anaesth. 2005;94(5):642-8.

13. White PF. The role of non-opioid analgesic techniques in the management of pain after ambulatory surgery. Anesth Analg. 2002;94(3):577-85.

14. Sinatra RS, Jahr JS, Reynolds LW, Visusi ER, Groudine SB, Payen-Champenois C. Efficacy and safety of single and repeated administration of 1 gram intravenous acetaminophen injection (paracetamol) for pain management after major orthopedic surgery. Anesthesiology. 2005;102(4):822-31.

15. Hirate J, Zhu CY, Horikoshi I, Bhargava VO. First-pass metabolism of acetaminophen in rats after low and high doses. Biopharm Drug Dispos. 1990;11(3):245-52.

16. Lin JH, Chiba M, Baillie TA. Is the role of the small intestine in first-pass metabolism overemphasized? Pharmacol Rev. 1999;51(2):135-58.

17. Kumpulainen E, Kokki H, Halonen T, Heikkinen M, Savolainen J, Laisalmi M. Paracetamol (acetaminophen) penetrates readily into the cerebrospinal fluid of children after intravenous administration. Pediatrics. 2007; 119(4):766-71.

18. Gregoire N, Hovsepian L, Gualano V, Evene E, Dufour G, Gendron A. Safety and pharmacokinetics of paracetamol following intravenous administration of $5 \mathrm{~g}$ during the first $24 \mathrm{~h}$ with a 2 -g starting dose. Clin Pharmacol Ther. 2007;81(3):401-5.

19. Yeh YC, Reddy P. Clinical and economic evidence for intravenous acetaminophen. Pharmacotherapy. 2012;32(6):559-79.

20. Higgins JPT, Altman DG, Gøtzsche PC, Jüni P, Moher D, Oxman AD, et al. The Cochrane Collaboration's tool for assessing risk of bias in randomised trials. BMJ. 2011;343:d5928.

21. Ameer B, Divoll M, Abernethy DR, Greenblatt DJ, Shargel L. Absolute and relative bioavailability of oral acetaminophen preparations. J Pharm Sci. 1983;72(8):955-8. 
22. Pettersson PH, Jakobsson J, Owall A. Intravenous acetaminophen reduced the use of opioids compared with oral administration after coronary artery bypass grafting. J Cardiothorac Vasc Anesth. 2005;19(3):306-9.

23. Van der Westhuizen J, Kuo PY, Reed PW, Holder K. Randomised controlled trial comparing oral and intravenous paracetamol (acetaminophen) plasma levels when given as preoperative analgesia. Anaesth Intensive Care. 2011;39(2):242-6.

24. Brett CN, Barnett SG, Pearson J. Postoperative plasma paracetamol levels following oral or intravenous paracetamol administration: a double-blind randomised controlled trial. Anaesth Intensive Care. 2012;40(1):166-71.

25. Singla NK, Parulan C, Samson R, Hutchinson J, Bushnell R, Beja EG, et al. Plasma and cerebrospinal fluid pharmacokinetic parameters after singledose administration of intravenous, oral, or rectal acetaminophen. Pain Pract. 2012;12(7):523-32.

26. Fenlon S, Collyer J, Giles J, Bidd H, Lees M, Nicholson J, et al. Oral vs intravenous paracetamol for lower third molar extractions under general anaesthesia: is oral administration inferior? Br J Anaesth. 2013;110(3): 432-7.

27. McNicol ED, Tzortzopoulou A, Cepeda MS, Francia MB, Farhat T, Schumann R. Single-dose intravenous paracetamol or propacetamol for prevention or treatment of postoperative pain: a systematic review and meta-analysis. Br J Anaesth. 2011;106(6):764-75.

28. Rawlins MD, Henderson DB, Hijab AR. Pharmacokinetics of paracetamol (acetaminophen) after intravenous and oral administration. Eur J Clin Pharmacol. 1977;11(4):283-6.
Farah Jibril, BSc(Pharm), PharmD, is a Clinical Pharmacist at the National Center for Cancer Care and Research, Doha, Qatar.

Sherif Sharaby, BSc(Pharm), is a Pharmacist with the San Joaquin Valley Rehabilitation Hospital, Fresno, California.

Ahmed Mohamed, BSc(Pharm), PhD, is an Assistant Professor with the College of Pharmacy, Qatar University, Doha, Qatar.

Kyle J Wilby, BSP, ACPR, PharmD, is an Assistant Professor - Clinical Pharmacy and Practice, College of Pharmacy, Qatar University, Doha, Qatar. Competing interests: None declared.

Address correspondence to:

Dr Kyle J Wilby

College of Pharmacy

Qatar University

PO Box 2713

Doha, Qatar

e-mail: kjw@qu.edu.qa

Funding: None received.

\section{CORRECTION \\ Measuring Anti-Factor Xa Activity to Monitor Low-Molecular-Weight Heparin in Obesity: A Critical Review - Correction}

In a critical review of the literature concerning measurement of anti-factor Xa activity for the purpose of monitoring low-molecular-weight heparin (LMWH) in patients with obesity, two numeric errors appeared, both in relation to manufacturer information for tinzaparin. The correct values are presented here.

In Table 2, which presents the manufacturers' published peak anti-factor Xa concentrations with various LMWH regimens, the third regimen for tinzaparin, as shown in the last row of the table, should be $175 \mathrm{U} / \mathrm{kg}$ (not $150 \mathrm{U} / \mathrm{kg}$ ).

In the section "Are the Pharmacokinetic Parameters Unpredictable in Patients with Obesity, Because of Either
Intrinsic Variability or the Presence of Other Confounding Factors?" (page 43), the dosage cap for tinzaparin should be $18000 \mathrm{U}$ daily, not $28000 \mathrm{U}$ daily. The first two sentences of this section are reproduced here, for context:

The product monographs recommend caution in weightbased dosing for patients with body weight over $120 \mathrm{~kg}$ (enoxaparin), ${ }_{17}$ over $90 \mathrm{~kg}$ (dalteparin), ${ }^{18}$ or over $105 \mathrm{~kg}$ (tinzaparin). ${ }^{19}$ They also recommend capping the dosage of enoxaparin at $150 \mathrm{mg}$ twice daily or $210 \mathrm{mg}$ once daily, ${ }^{17}$ dalteparin at $18000 \mathrm{U}$ daily, ${ }^{18}$ and tinzaparin at 18000 U daily. ${ }^{19}$

\section{Reference}

1. Egan G, Ensom MHH. Measuring anti-factor Xa activity to monitor lowmolecular-weight heparin in obesity: a critical review. Can J Hosp Pharm. 2014;68(1):33-47. 